\title{
Quantum Circuit Controller
}

This paper was downloaded from TechRxiv (https://www.techrxiv.org).

\section{LICENSE}

CC BY-NC-SA 4.0

SUBMISSION DATE / POSTED DATE

$18-01-2022$ / 24-01-2022

CITATION

Delgado, Alberto (2022): Quantum Circuit Controller. TechRxiv. Preprint. https://doi.org/10.36227/techrxiv.18651629.v1

$\mathrm{DOI}$

10.36227/techrxiv.18651629.v1 


\title{
Quantum Circuit Controller
}

\author{
Alberto Delgado $^{1}$, IEEE Senior Member
}

\begin{abstract}
A quantum circuit controller, parametrized quantum circuit and operator, is used in a closed loop system to control a nonlinear plant. The controller has eight parameters, the first four can be fixed beforehand (set point and control surface smoothness), the remaining four are tuned manually using a set of rules in natural language.
\end{abstract}

\section{INTRODUCTION}

Controllers for closed loop systems can be designed using rules formulated in natural language, this approach is known as fuzzy logic control [1].

On the other hand, research on quantum computers, as the next step in computation, has solid theoretical foundations [2]. Algorithms in quantum computers are represented by a quantum circuit, that is, a sequence of unitary operations from an initial superposition of quantum states to a final superposition where the result can be measured with certain probability.

Quantum circuits can also be used as parametrized nonlinear functions or quantum parametric models, here probabilities are a function of independent variables and parameters to be tuned or learned with optimization techniques.

This paper illustrates an application of a parametrized quantum model to control a nonlinear plant in a closed loop. The expectation value of a diagonal operator, using a parametrized quantum circuit, results in a quantum controller, a nonlinear function $\mathrm{u}$ of the closed loop error $e$, its derivative $\dot{e}$, and eight tunable parameters $\left(e_{0}, \Delta e_{0}, s_{1}, s_{2}, g_{1}, g_{2}, g_{3}, g_{4}\right)$.

The paper is organized as follows, section two presents the nonlinear plant and the closed loop control problem. Section three introduces the quantum circuit controller, quantum circuit and operator. Section four formulates the rules in natural language to control the nonlinear plant. Section five shows the control surface generated by the quantum controller for two groups of parameters. Finally conclusions are formulated in section six.

\section{NONLINEAR CONTROL}

Consider the nonlinear plant [3] shown in figure 1, the desired position for the pendulum is $\theta_{r}$ and the actual position is $\theta$. The control input $\mathrm{u}$ is positive in the counterclockwise direction.

To control the nonlinear plant the closed loop shown in figure 2 is proposed, when the parameters are fixed the Quantum Circuit Controller (QCC) is a nonlinear function of the closed loop error $e$ and its derivative $\dot{e}$,

\footnotetext{
${ }^{1}$ Alberto Delgado is with the Electrical and Electronics Engineering Department, National University of Colombia, Bogota, CO adelgadodieee.org
}

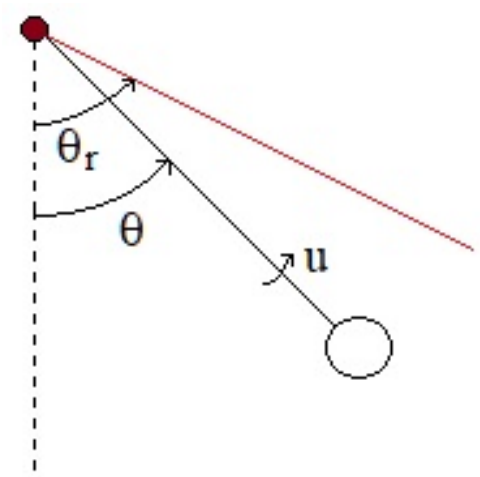

Fig. 1. Nonlinear plant, pendulum or single link manipulator, $\theta_{r}$ is the reference position, $\theta$ is the actual position, and $\mathrm{u}$ is the control input. Note that the error, $e=\theta_{r}-\theta$, is positive when the pendulum is below the reference, $\mathrm{u}$ is positive in the indicated direction.

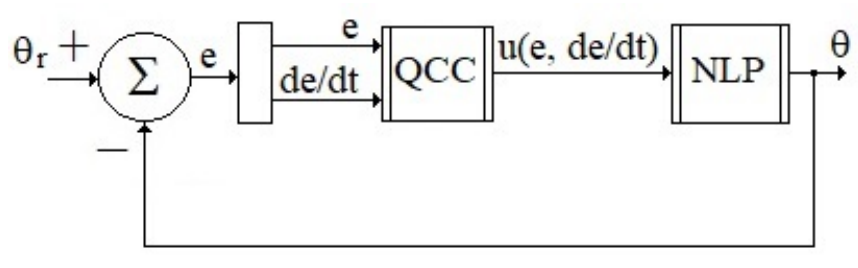

Fig. 2. Closed loop with a nonlinear controller, $u(e, \dot{e})$ and a nonlinear plant NLP, the error $e=\theta_{r}-\theta$.

In continuous time, the closed loop error and its derivative follow the equations,

$$
\begin{aligned}
e(t) & =\theta_{r}-\theta(t) \\
\dot{e} & =\frac{d e(t)}{d t}
\end{aligned}
$$

In discrete time, for the digital implementation,

$$
\begin{aligned}
e(k) & =\theta_{r}-\theta(k) \\
\Delta e(k) & =e(k)-e(k-1)
\end{aligned}
$$

where $\mathrm{k}$ is the sample number.

\section{QUANTUM CIRCUIT CONTROLLER}

The controller has two components, a parametrized quantum circuit with two qubits, the first qubit state depends on the closed loop error $e$ and the second qubit state depends on $\Delta e$. 


$$
\begin{aligned}
\mid \psi_{1}(e)> & =\left[\begin{array}{l}
\cos \alpha_{1}(e) \\
\sin \alpha_{1}(e)
\end{array}\right] \\
\mid \psi_{2}(\Delta e)> & =\left[\begin{array}{l}
\cos \alpha_{2}(\Delta e) \\
\sin \alpha_{2}(\Delta e)
\end{array}\right],
\end{aligned}
$$

The arguments $\left\{\alpha_{1}, \alpha_{2}\right\}$ are functions of the closed loop error and its time change $\{e, \Delta e\}$ with parameters $\left\{e_{0}, \Delta e_{0}, s_{1}, s_{2}\right\}$,

$$
\begin{aligned}
\alpha_{1}(e) & =\frac{\pi / 2}{1+\exp \left[-\left(e-e_{0}\right) / s_{1}\right]} \\
\alpha_{2}(\Delta e) & =\frac{\pi / 2}{1+\exp \left[-\left(\Delta e-\Delta e_{0}\right) / s_{2}\right]} .
\end{aligned}
$$

The second controller component is an operator $\mathrm{G}$ with real values or gains for the $(e, \Delta e)$ controller,

$$
G=\left[\begin{array}{cccc}
g_{1} & 0 & 0 & 0 \\
0 & g_{2} & 0 & 0 \\
0 & 0 & g_{3} & 0 \\
0 & 0 & 0 & g_{4}
\end{array}\right]
$$

The resulting state $\mid \psi(e, \Delta e)>$ of this quantum controller is the tensor product of the individual states,

$$
\begin{aligned}
\mid \psi(e, \Delta e)> & =\mid \psi_{1}(e) \psi_{2}(\Delta e)> \\
\mid \psi(e, \Delta e)> & =\left[\begin{array}{l}
\cos \alpha_{1}(e) \cos \alpha_{2}(\Delta e) \\
\cos \alpha_{1}(e) \sin \alpha_{2}(\Delta e) \\
\sin \alpha_{1}(e) \cos \alpha_{2}(\Delta e) \\
\sin \alpha_{1}(e) \sin \alpha_{2}(\Delta e)
\end{array}\right]
\end{aligned}
$$

The expectation value of operator $G$, quantum circuit controller, is a parametrized nonlinear function of the closed loop error and its time change,

$$
\begin{aligned}
u(e, \Delta e)= & <\psi(e, \Delta e)|G| \psi(e, \Delta e)> \\
u(e, \Delta e)= & g_{1}\left[\cos \alpha_{1}(e) \cos \alpha_{2}(\Delta e)\right]^{2} \\
& +g_{2}\left[\cos \alpha_{1}(e) \sin \alpha_{2}(\Delta e)\right]^{2} \\
& +g_{3}\left[\sin \alpha_{1}(e) \cos \alpha_{2}(\Delta e)\right]^{2} \\
& +g_{4}\left[\sin \alpha_{1}(e) \sin \alpha_{2}(\Delta e)\right]^{2}
\end{aligned}
$$

It is important to mention that (7) is a continuous map from $\{e, \Delta e\}$ to $u(e, \Delta e)$, passing through the discrete state space of the quantum system (3). In total the quantum circuit controller has eight parameters $\left\{e_{0}, \Delta e_{0}, s_{1}, s_{2}, g_{1}, g_{2}, g_{3}, g_{4}\right\}$, the first four change the state probabilities and the others can be seen as controller gains.

\section{RULES}

Controllers can be designed using rules in natural language, these are called fuzzy logic controllers [1]. A set of rules to control the pendulum, figure 1 , are formulated in tabular form as shown in Table 1 where $\mathrm{P}$ means positive, $P B$ positive big, $\mathrm{N}$ negative, $N B$ negative big.

For example, when the error is $\mathrm{P}$ means that the pendulum is below the reference (2). If the pendulum is moving clockwise, the error change $\Delta e$ is $\mathrm{N}$ (2). In this case, the pendulum is moving away from the reference, clockwise direction, so the input $\mathrm{u}$ needs to be a big positive number $P B$ for $\theta$ to get closer to the reference. When the pendulum is moving in the counterclockwise direction, $\Delta e$ is $\mathrm{P}(2)$, then the control input is just a positive number $\mathrm{P}$ because $\theta$ is closer to the reference.

In the situation when the pendulum is above the reference, negative error, and moving in the counterclockwise direction, the control input needs to be $N B$. Finally, if the pendulum moves in the clockwise direction, the input is $\mathrm{N}$.

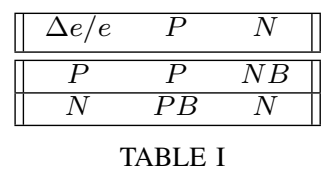

QuANTUM CONTROLler Rules.

\section{SIMULATIONS}

After formulating the rules in natural language to control the pendulum (reduce the close loop error $e$ and its time change $\Delta e$ ), the parameters of the quantum circuit controller are tuned to satisfy Table 1 .

Parameters $e_{0}, \Delta e_{0}$ are set to zero, the desired closed loop error and its time change are zero, $\theta_{r}=\theta$ and $\Delta e=0$. Parameters $s_{1}, s_{2}$ control the surface $u(e, \Delta e)$ smoothness, small values produce discontinuities. Parameters $g_{1}, g_{2}, g_{3}, g_{4}$ are the controller gains, these are tuned to follow the natural language rules, Table 1.

Two simulations illustrate the control surface $u(e, \Delta u)$ according to the formulated rules, Table 2 shows the parameter values of figures 3 and 4 .

Numerical values for the gains $\left(g_{1}, g_{2}, g_{3}, g_{4}\right)$ are chosen according to the linguistic values (NB, N, P, PB) from the rules, however further numerical adjustments, fine tuning, can be made when the quantum circuit controller is operating inside the closed loop and time response characteristics are considered in the overall design.

\begin{tabular}{||ccccccccc||}
\hline fig. & $e_{0}$ & $\Delta e_{0}$ & $s_{1}$ & $s_{2}$ & $g_{1}$ & $g_{2}$ & $g_{3}$ & $g_{4}$ \\
\hline \hline 3 & 0.0 & 0.0 & 0.1 & 0.1 & -1.0 & 2.0 & -2.0 & 1.0 \\
\hline 4 & 0.0 & 0.0 & 0.5 & 0.5 & -1.0 & 2.0 & -2.0 & 1.0 \\
\hline
\end{tabular}

TABLE II

PARAMETERS QUANTUM CONTROLLER.

Note that formula (7) is equivalent to,

$$
\begin{aligned}
& u(e, \dot{e})=g_{1} p_{1}+g_{2} p_{2}+g_{3} p_{3}+g_{4} p_{4} \\
& u(e, \dot{e})=\sum_{i=1}^{n} g_{i} p_{i}\left(e, \Delta e, e_{0}, \Delta e_{0}, s_{1}, s_{2}\right) .
\end{aligned}
$$

where $p_{i}\left(e, \Delta e, e_{0}, \Delta e_{0}, s_{1}, s_{2}\right)$ is the probability of gain $g_{i}, \mathrm{i}=1,2, \ldots, \mathrm{n}$, two qubits $\mathrm{n}=4$. Once the parameters are fixed probabilities depend only on $(e, \Delta e)$, big positive values for $(e, \Delta e)$ result in $p_{1} \rightarrow 0.0, p_{4} \rightarrow 1.0$, big negative values for $(e, \Delta e)$ result in $p_{1} \rightarrow 1.0, p_{4} \rightarrow 0.0$. When $e=$ $0, \Delta e=0$, all probabilities are the same. 


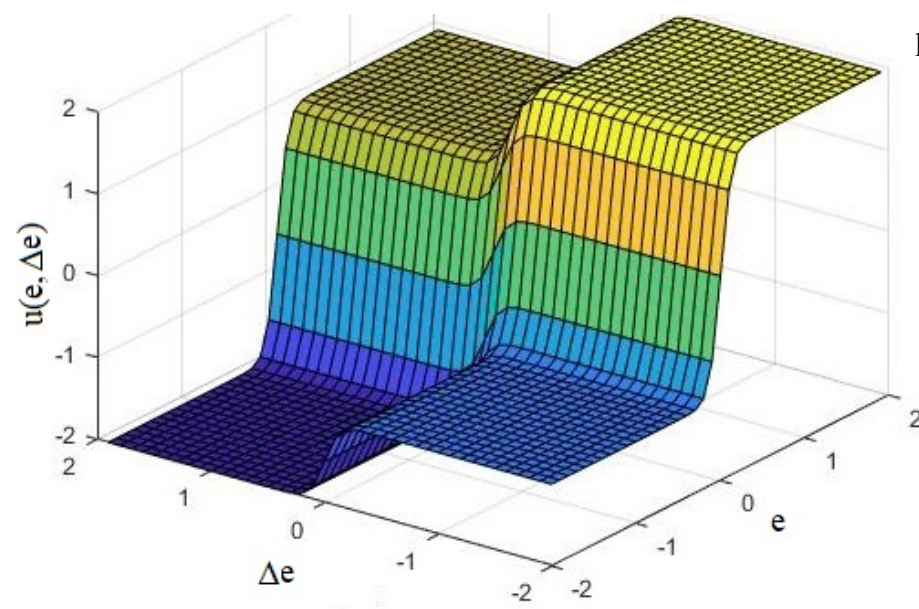

his research.

\section{REFERENCES}

[1] J.M. Mendel, Fuzzy Logic Systems for Engineering: A Tutorial, Proc. IEEE, Vol. 83, No. 3, pp. 345 - 377, 1995.

[2] M.A. Nielsen, and I.L. Chuang, Quantum Computation and Quantum Information. Cambridge: Cambridge University Press, 2010.

[3] H.K. Khalil, Nonlinear Systems. Macmillan Publishing Company, Singapore, 1992

[4] A. Delgado, "Quantum memristor," TechRxiv.12489149.v2, 2020.

Fig. 3. Control surface $\mathrm{u}$ as function of $e$ and $\Delta e$, the parameters $s_{1}, s_{2}$ are small so the surface has discontinuities.

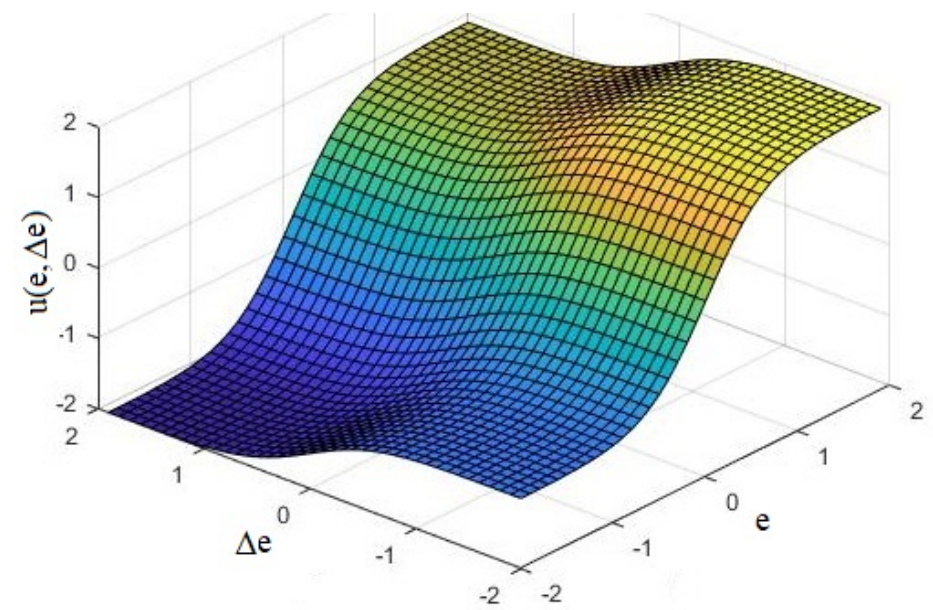

Fig. 4. Control surface $\mathrm{u}$ as function of $e$ and $\Delta e$, the parameters $s_{1}, s_{2}$ are increased so the surface is smoother.

\section{CONCLUSIONS}

A quantum circuit controller, parametrized quantum circuit and operator, was presented. In total there are eight parameters, four belong to the quantum circuit (probabilities) and the other four belong to the operator (controller gains).

When the parameters of the quantum circuit are fixed, the state probabilities are function of the closed loop error $e$ and its time change $\Delta e$. The natural language rules, Table 1 , guide the manual tuning process of the controller gains $\left(g_{1}, g_{2}, g_{3}, g_{4}\right)$ with numerical values.

Finally, in a general case, the quantum circuit controller parameters $\left(e_{0}, \Delta e_{0}, s_{1}, s_{2}, g_{1}, g_{2}, g_{3}, g_{4}\right)$ can be tuned automatically by using well known optimization techniques already available in the literature. Note that some parameters are known beforehand and this reduces the search time.

\section{ACKNOWLEDGMENT}

The author, Alberto Delgado, thanks his employer The National University of Colombia at Bogota for supporting 